\title{
Effect of concentration and dispersion of filler fractions on adhesive strength and abrasion resistance of an epoxy and sand composite
}

\author{
Alexander Mikhalchenkov ${ }^{*}$, Marina Mikhalchenkova ${ }^{1}$, Sergey Feskov ${ }^{1}$ and Alexandr \\ Kononenko ${ }^{2}$ \\ ${ }^{1}$ Bryansk state agrarian University, 2A, Soviet Str., the village of Kokino, Vygonichskogo district, \\ Bryansk region, 243365, Russian Federation \\ ${ }^{2}$ Bauman Moscow State Technical University, 5-1, 2-ya Baumanskaya Str., Moscow, 105005, \\ Russian Federation
}

\begin{abstract}
The increase in the adhesive strength of the epoxy and sand composite is equally regulated by the content of the sand component and the dispersiveness of its particles. The wear resistance of the composite is mainly determined by the size of sand particles, and the concentration significantly affects the degree of resistance to abrasive wear only when the fraction dispersiveness is equal to $1 \mathrm{~mm}$. The maximum adhesive strength and wear resistance are characteristic for a composite of the following composition: 30 pts. wt. epoxy glue and 70 pts. wt. natural sand with a dispersiveness of $1 \mathrm{~mm}$.
\end{abstract}

\section{Introduction}

Elimination of worn spots of non-traditional shape, as well as improving the operational properties of the working surfaces of parts using adhesive polymer composites has recently become increasingly wide [1,2]. At the same time, based on the working conditions of the friction surfaces, composites with various properties or their combination are required, which makes it necessary to conduct full-scale experiments to select the ideal compositions. The determining parameters of such substances are adhesive strength and resistance to various types of wear, including abrasive one.

Therefore, the aim of the study was to identify the degree of influence of the composition of the composite with the epoxy matrix and the dispersiveness of sand filler on its adhesive strength and resistance to abrasive wear.Error! Reference source not found..

\section{Materials and Research Methods}

During the tests, we used a material consisting of epoxy glue (epoxy and diane resin ED-20, 100 weight parts (pts. wt.); polyethylene polyamine hardener, 10 pt wt.; and filler, natural quartz sand. The abrasion resistance of five compositions of E/S composites (pts. wt.) was

\footnotetext{
*Corresponding author: mihalchenkov.alexandr@yandex.ru
} 
studied: 30/70; 40/60; 50/50; 60/40 and 70/30, as well as adhesive strength of six compositions - E/S (pts. wt.): 30/70; 40/60; 50/50; 60/40, 70/30, and 80/20. The numerator indicates the amount of epoxy glue, $E$, the denominator indicates the amount of sand filler $\mathrm{P}$. The dispersiveness (de) of the sand component was: $0.10,0.25,0.50$, and $1.00 \mathrm{~mm}$, which meets the definition of sand as a component of the soil medium [3]. Sands with the indicated fraction sizes were used in the preparation of each composition. Thus, the tested number of composites, taking into account variations in the concentration and dispersiveness of filler particles, amounted to 24 units in total.

The adhesive strength of the "adhesive polymer composite/substrate" system was determined by the in-house method (Fig. 1) [4] and assessed by the shear stress $\left(\tau_{C}\right)$. The testing technique, in this case, consists in forcing through the cured composite 3 formed in the inner cavity of the metal matrix 1 using the punch pin 2 with the help of the power mechanism with load fixing providing shear (Fig. 2). This method allows avoiding process difficulties in the preparation of prototypes and ensuring high confidence of the data being obtained due to the close relevance of the experiment to the actual operating conditions of the coating.

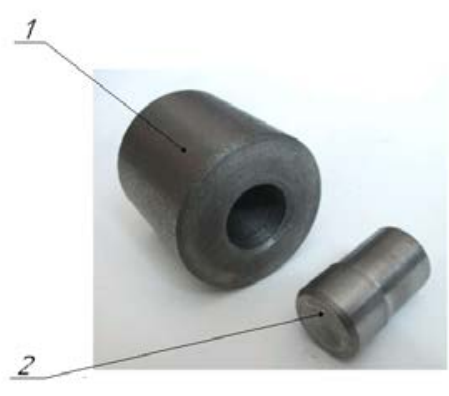

a)

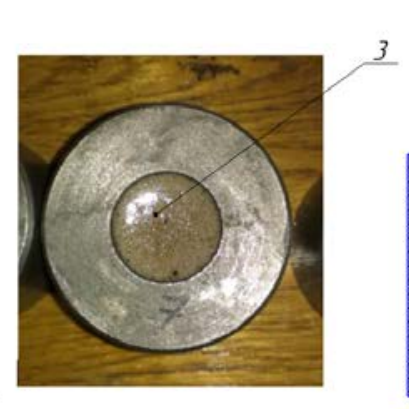

b)

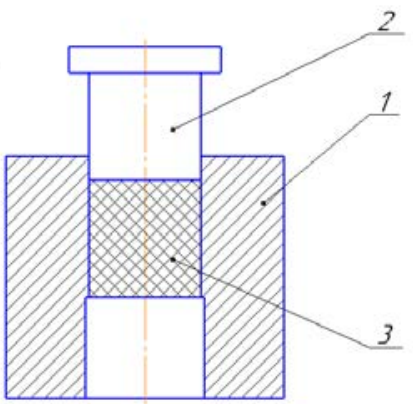

c)

Fig. 1. Adhesive strength test fixture (a, b - full-scale specimens, c - test fixture diagram): 1 - matrix, 2 - punch pin (rod), 3 - coating material

Experiments were carried out on MI20UMT automated test complex equipped with a PC, which allows tracking the features of the loading process and directly record the "load on the composite-displacement of the punch pin" diagram on the monitor.

Studies of the abrasion resistance of various composite compositions were also carried out according to the method developed by the authors, which allows testing several polymer composite materials with various properties simultaneously and under the same conditions in a non-rigid abrasive (Fig. 2) [5]. The method is versatile and may be extended to testing materials of a different nature; moreover, it is possible to change the speed of prototypes movement and the composition of the wear medium in a wide range. As an abrasive medium, a mixture of the following is used: 40 pts. wt. of natural sand and 60 pts. wt. of gravel chips with a diameter of 6-10 $\mathrm{mm}$ when rotating at a speed of $1000 \mathrm{~min}^{-1}$. 


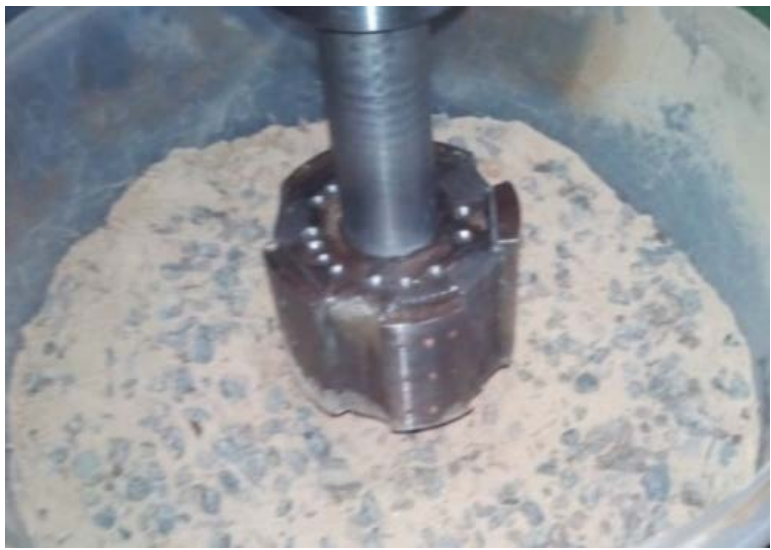

Fig. 2. Fixture and abrasive medium at the time of testing

\section{Results and Discussion}

The obtained experimental data on the effect of the concentration of the component and the dispersiveness of sand on the adhesive strength indicate the complex nature of the change in this parameter with increasing dispersiveness and the amount of sand filler (Fig. 3).

An increase in the amount of the sand component leads to a rather dramatic increase in the adhesion strength $\tau_{c}$ (Fig. 3) and it is explained by an increase in the area of contact of particles with the surface of the substrate. It should be noted that such an effect of sand fractions takes place with a simultaneous increase in the amount of the sand component in the composite and an increase in the size of its particles. The adhesive strength of the adhesive mass without filler is about $10.0 \mathrm{MPa}$, while the presence of sand in an amount of 60-70 pts. wt. provides $\tau_{c}=19.0 \mathrm{MPa}$. That is, $\tau_{\mathrm{c}}$ almost doubles.

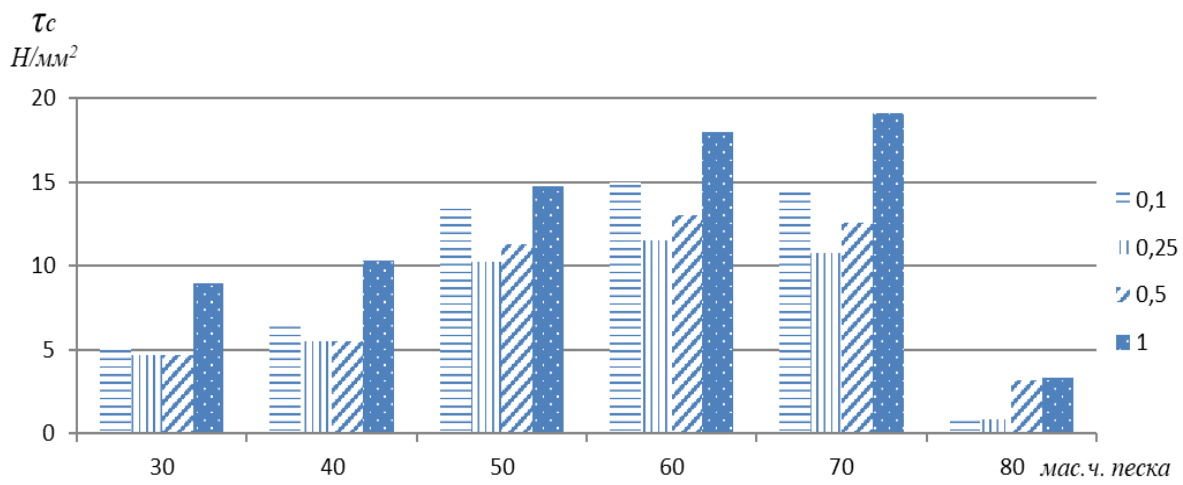

Fig. 3. The diagram of dependencies of the adhesive strength on the dispersiveness of sand particles and its concentration in the composite $(0.1,0.25,0.5$, and 1 are particle sizes in $\mathrm{mm})$

After that, with an increase in the sand concentration ( $k$ pts. wt.) of more than $70 \%$, a small amount of glue is no longer able to bind its particles into a monolith and the adhesive strength of the material as a whole decreases due to a break of the internal adhesion of filler particles in the epoxy matrix.

It is known that the adhesion strength of the composite to the substrate is determined by two factors: the adhesive properties of the epoxy composition and sand particles, which resist 
the detachment due to the friction against the substrate. Given that the adhesive strength being created by the epoxy matrix is approximately constant for each composition, the value of $\tau_{c}$ will be mainly determined by the dispersiveness of filler particles and its content in the composite and it is expressed by the equation:

$$
\tau_{c}=3,45+0,21 k+4,7 d_{\ni} .
$$

In accordance with formula 1 , coefficients for $k$ and $d_{e}$ actually differ by an order of magnitude. However, the concentration values (assessed by the amount of sand in the material) and the dispersiveness values of filler particles also differ by an order of magnitude, therefore, $k$ and $d_{e}$ to the equal extent affect the adhesive strength of the composite and metal substrate system (The results obtained for $k=80$ pts. wt. mathematical processing were excluded as non-characteristic).

Another factor influencing $\tau_{c}$ will be the static friction between the filler particles and the metal substrate, which is not constant and depends on the size of filler particles. It will be high when sand particles are large enough. For large fractions, the total contact area will be relatively small, but, penetrating into the substrate and penetrating relatively deeply into it, they create greater static friction and, consequently, an increase in adhesive strength.

In addition, the mechanism of movement of the formed material along the metal substrate may be compared to a certain extent with the contacting of surfaces with uneven roughness due to different sizes of sand particles. In this case, the friction factor is significant with a high roughness, therefore, adhesion will be increased as well.

As a result of the experiments conducted, it was found that the adhesion strength of the adhesive-sand composite, depending on the particle size and concentration of components, is of complex nature being explained not only by the adhesive capacity of the matrix but also by the interaction of sand fractions with the surface of the substrate, as well as the structure of the composite. The maximum values of adhesive strength are compositions with a natural sand content of 60-70 pts. wt. and particle diameters of approximately $1 \mathrm{~mm}$ (Fig. 3).

The concentration of components of the composite and the dispersiveness of filler also introduce certain peculiarities into the wear mechanics.

It should be assumed that the wear resistance of the composite will be determined by two factors: tribotechnical and adhesive properties of the epoxy composition, as well as the size of sand particles that resist wearing due to the friction against the abrasive medium.

Considering that the noted properties of the epoxy matrix are approximately constant for each composition, the value of the wear resistance will be limited mainly by the dispersiveness of filler and its content in the composite. The static friction between filler particles and the abrasive medium will be high either when particles are very small or when they are large. In the presence of small particles, there is a large total area of their contact with the abrasive medium, which means that the static friction between them is quite high. For large particles, this area will be relatively small, but large particles, contacting with particles of the medium and penetrating relatively deeply into it, also create high static friction. Thus, there are particles of such a size that create minimal static friction, providing minimal wear.

It should be especially noted that the theory above is characteristic exclusively for the local contact of the abrasive medium and sand particles that are part of the composite. In this regard, a discrepancy between the resistance to abrasive wear obtained in real conditions and in laboratory conditions is possible.

Another wear mechanism is also possible. The movement of the composite along the abrasive medium to a certain extent may be compared to the contact of surfaces of different roughness. Moreover, the coefficient of friction is large with high roughness, and it is also large for polished surfaces due to the large area of their contact. This is confirmed by the fact that an increase in dispersiveness leads to a decrease in the wear resistance. 
In addition, the internal adhesion between the larger particles and the epoxy component will be slightly increased in comparison with the fine fractions due to their larger contact area. In turn, this circumstance makes it difficult to "tear" particles from the total mass of the composite when the coatings are in contact with an abrasive medium.

At the same time, an excessive increase in the sand component in the material will lead to a break of the adhesive strength between sand particles and the adhesive mass, thereby reducing the resistance to "tearing" of anti-abrasive quartz particles, and, as a consequence, to an increase in the wear rate.

As follows from the diagrams (Fig. 4), the maximum wear resistance of the adhesive polymer epoxy and sand composite is achieved with the following parameters: composition - 70 (60) pts. wt. of the sand component and 30 (40) pts. wt. of the epoxy glue; dispersiveness of sand particles - $1 \mathrm{~mm}$, which confirms the suggestions above.

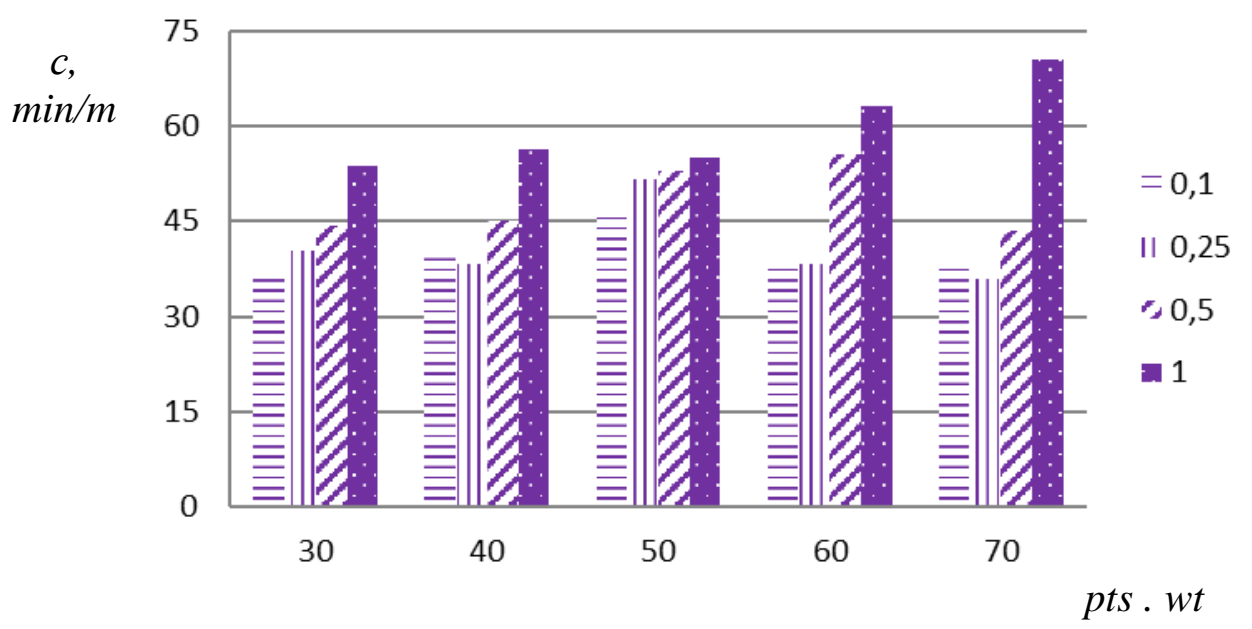

Fig. 4. Diagrams of the wear resistance of various compositions of the composite, taking into account the dispersiveness of sand $(0.1,0.25,0.5$, or 1 - particle sizes in $\mathrm{mm})$

At the same time, a change in the quantitative ratio of components introduces certain features into the wear mechanics.

According to the data obtained, an increase in the amount of the sand component in the composite has a different effect on the abrasion resistance (Fig. 5) and is directly related to the particle size of sand filler (The construction of the diagrams was carried out according to the results when the wear occurred in the steady state).

In the case where $d_{e}=0.1 \mathrm{~mm}$, an increase in the amount of sand in the substance does not actually affect the wear resistance (Fig. 5 straight line 1).

The effect of the concentration on the growth of $c$ for $d_{e}=0.25$ and $0.5 \mathrm{~mm}$ is more obvious (Fig. 5, curves 2 and 3). At the same time, there is an increase in the wear resistance, although insignificant. The most significant effect of the concentration change on the wear resistance is manifested when sand with the particle size of approximately $1.0 \mathrm{~mm}$ acts as a dispersed filler (Fig. 3, curve 4). 


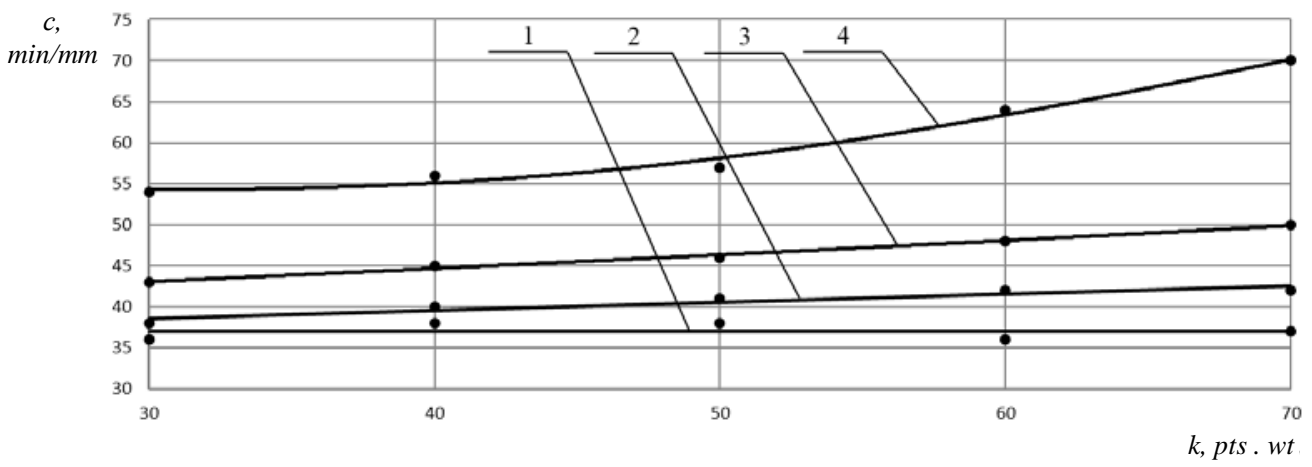

Fig. 5. Dependence of the resistance to the abrasive wear $c$ on the concentration $k$ (pts. wt.) of sand in the composite with dispersiveness values $d_{e}: 1-0.1 \mathrm{~mm}, 2-0.25 \mathrm{~mm}, 3-0.5 \mathrm{~mm}, 4-1.0 \mathrm{~mm}$

The regression equation (2) obtained by the mathematical processing of the experimental data, connecting the resistance to abrasive wear $(c)$, the concentration of components $(k)$, and the dispersiveness of filler particles $\left(d_{e}\right)$ shows that the size of sand particles has the most significant effect on the abrasion resistance.

$$
c=31,8+0,098 k+22,25 d_{3}
$$

\section{Outcomes}

1. The adhesive strength of the epoxy and sand composite is approximately equally regulated by the content of the sand component and the dispersiveness of its particles and it increases with an increase in these parameters.

2. The size of sand particles has a decisive influence on the resistance of the composite to the abrasive wear. The influence of the concentration of components significantly affects the value of $c$ only when the dispersiveness of fractions is equal to $1 \mathrm{~mm}$.

3. A composition that is a polymer self-hardening base consisting of 30 pts. wt. of the epoxy adhesive with a filler of 70 pts. wt. of natural sand with a particle size of $1 \mathrm{~mm}$ is ideal from the point of view of the adhesive strength and wear resistance.

\section{References}

1. Li R.I., Kirsanov F.A., Kiba M.R. Technology and equipment for high-precision polymer restoration of fitment holes in automotive housing parts // Polymer Science. Series D. 2016. - T. 9. - № 3. - C. 312-316.

2. Kononenko A.S., Kildeyev T.A., Soloviova A.A. Features of restoration of spindle shafts of metal-cutting machines with polymer materials and nanocomposites based on them // Repair, Restoration, and Modernization. 2018. No.10. PP. 3-8.

3. Filin Yu.I. Epoxy composite for the increase of the operation life of heat-strengthened plowshares // Rural Mechanizer. - 2017. - No.5. - PP. 36-37.

4. Mikhalchenkov A.M., Feskov S.A., Mozheiko A.V., Smirnov A.Ye. Improving the technique for determining the adhesive strength of adhesive polymer composites // Bulletin of the Bryansk State Agricultural Academy. - 2019 .-- No.2 (72). - PP. 45-49.

5. Mikhalchenkov A.M., Biryulina Ya.Yu., Filin Yu.I. The evolution of accelerated comparative tests of adhesive polymer-dispersed composites for the abrasion resistance // Repair. Recovery. Modernization. - 2016. - No.4. - PP. 41-43. 